\title{
On improvements of the Rozanova's inequality
}

\author{
Chang-Jian Zhao ${ }^{{ }^{*}}$ and Wing-Sum Cheung ${ }^{2}$
}

* Correspondence: chjzhao@163. com

${ }^{1}$ Department of Mathematics, China Jiliang University, Hangzhou 310018, China

Full list of author information is available at the end of the article

\section{Abstract}

In the present paper, we establish some new Rozanova's type integral inequalities involving higher-order partial derivatives. The results in special cases yield some of the interrelated results on Rozanova's inequality and provide new estimates on inequalities of this type.

MS (2000) Subject Classifiication: $26 D 15$.

Keywords: Opial's inequality, Hölder's inequality, Rozanova's inequality

\section{Introduction}

In the year 1960, Opial [1] established the following integral inequality:

Theorem A Suppose $f \in C^{1}[0, h]$ satisfies $f(0)=f(h)=0$ and $f(x)>0$ for all $x \in(0, h)$. Then

$$
\int_{0}^{h}\left|f(x) f^{\prime}(x)\right| d x \leq \frac{h}{4} \int_{0}^{h}\left(f^{\prime}(x)\right)^{2} d x .
$$

The first Opial's type inequality was established by Willett [2] as follows:

Theorem B Let $x(t)$ be absolutely continuous in $[0, a]$, and $x(0)=0$. Then

$$
\int_{0}^{a}\left|x(t) x^{\prime}(t)\right| d t \leq \frac{a}{2} \int_{0}^{a}\left|x^{\prime}(t)\right|^{2} d t
$$

A non-trivial generalization of Theorem B was established by Hua [3] as follows:

Theorem C Let $x(t)$ be absolutely continuous in $[0, a]$, and $x(0)=0$. Futher, let $l$ be a positive integer. Then

$$
\int_{0}^{a}\left|x(t) x^{\prime}(t)\right| d t \leq \frac{a^{l}}{l+1} \int_{0}^{a}\left|x^{\prime}(t)\right|^{l+1} d t .
$$

A sharper inequality was established by Godunova [4] as follows:

Theorem D Let $f(t)$ be convex and increasing functions on $[0, \infty)$ with $f(0)=0$. Further, let $x(t)$ be absolutely continuous on $[0, \tau]$, and $x(\alpha)=0$. Then, following inequality holds

$$
\int_{\alpha}^{\tau} f^{\prime}(|x(t)|)\left|x^{\prime}(t)\right| d t \leq f\left(\int_{\alpha}^{\tau}\left|x^{\prime}(t)\right| d t\right) .
$$

Rozanova [5] proved an extension of inequality (1.4) is embodied in the following:

Theorem $\mathbf{F}$ Let $f(t)$ and $g(t)$ be convex and increasing functions on $[0, \infty)$ with $f(0)=$ 0 , and let $p(t) \geq 0, p^{\prime}(t)>0, t \in[\alpha, a]$ with $p(\alpha)=0$. Further, let $x(t)$ be absolutely 
continuous on $[\alpha, a)$, and $x(\alpha)=0$. Then, following inequality holds

$$
\int_{\alpha}^{a} p^{\prime}(t) \cdot g\left(\frac{\left|x^{\prime}(t)\right|}{p^{\prime}(t)}\right) \cdot\left[f^{\prime}\left(p(t) \cdot g\left(\frac{|x(t)|}{p(t)}\right)\right)\right] d t \leq f\left(\int_{\alpha}^{a} p^{\prime}(t) \cdot g\left(\frac{\left|x^{\prime}(t)\right|}{p^{\prime}(t)}\right) d t\right) .
$$

The inequality (1.5) will be called as Rozanova's inequality in the paper.

Opial's inequality and its generalizations, extensions and discretizations play a fundamental role in establishing the existence and uniqueness of initial and boundary value problems for ordinary and partial differential equations as well as difference equations [6-13]. For Opial-type integral inequalities involving high-order partial derivatives, see $[14,15]$. For an extensive survey on these inequalities, see [16].

The first aim of the present paper is to establish the following Opial-type inequality involving higher-order partial derivatives, which is an extension of the Rozanova's inequality (1.5).

Theorem 1.1 Let $f$ and $g$ be convex and increasing functions on $[0, \infty)$ with $f(0)=0$, and let $p(s, t) \geq 0, D_{1} D_{2} p(s, t)=\frac{\partial^{2}}{\partial s \partial t} p(s, t), D_{1} D_{2} p(s, t)>0, s \in[\alpha, a], t \in[\beta, b]$ with $p$ $(s, \beta)=p(\alpha, t)=p(\alpha, \beta)=0$ and $\left.D_{1} D_{2} p(s, t)\right|_{t=\tau}=0$. Further, let $x(s, t)$ be absolutely continuous on $[\alpha, a) \times[\beta, b]$, and $x(s, \beta)=x(\alpha, t)=x(\alpha, \beta)=0$. Then following inequality holds

$$
\begin{gathered}
\int_{\alpha}^{a} \int_{\beta}^{b} D_{1} D_{2} p(s, t) \cdot g\left(\frac{\left|D_{1} D_{2} x(s, t)\right|}{D_{1} D_{2} p(s, t)}\right) \cdot \frac{\partial}{\partial t}\left[f\left(p(s, t) \cdot g\left(\frac{|x(s, t)|}{p(s, t)}\right)\right)\right] d s d t \\
\leq f\left(\int_{\alpha}^{a} \int_{\beta}^{b} D_{1} D_{2} p(s, t) \cdot g\left(\frac{\left|D_{1} D_{2} x(s, t)\right|}{D_{1} D_{2} p(s, t)}\right) d s d t\right) .
\end{gathered}
$$

We also prove the following Rozanova-type inequality involving higher-order partial derivatives.

Theorem 1.2 Assume that

(i) $f, g$ and $x(s, t)$ are as in Theorem 1.1,

(ii) $p(s, t)$ is increasing on $[0, a] \times[0, b]$ with $p(s, \beta)=p(\alpha, t)=p(\alpha, \beta)=0$,

(iii) $h$ is concave and increasing on $[0, \infty)$,

(iv) $\varphi(t)$ is increasing on $[0, a]$ with $\varphi(0)=0$,

(v) For $\gamma(s, t)=\int_{0}^{s} \int_{0}^{t} D_{1} D_{2} p(\sigma, \tau) g\left(\frac{\left|D_{1} D_{2} x(\sigma, \tau)\right|}{D_{1} D_{2} p(\sigma, \tau)}\right) d \sigma d \tau$,

$$
D_{1} D_{2} f(\gamma(s, t)) D_{1} D_{2} \gamma(s, t) \cdot \phi\left(\frac{1}{D_{1} D_{2} \gamma(s, t)}\right) \leq \frac{c_{(a, b)}}{\gamma(a, b)} \cdot \phi^{\prime}\left(\frac{t}{\gamma(a, b)}\right) .
$$

Then

$$
\begin{gathered}
\int_{0}^{a} \int_{0}^{b} D_{1} D_{2} f\left(p(s, t) g\left(\frac{|x(s, t)|}{p(s, t)}\right)\right) \cdot v\left(D_{1} D_{2} p(s, t) g\left(\frac{\left|D_{1} D_{2} x(s, t)\right|}{D_{1} D_{2} p(s, t)}\right)\right) d s d t \\
\leq w\left(\int_{0}^{a} \int_{0}^{b} D_{1} D_{2} p(s, t) g\left(\frac{|x(s, t)|}{D_{1} D_{2} p(s, t)}\right) d s d t\right)
\end{gathered}
$$


where

$$
\begin{aligned}
& v(z)=z h\left(\phi\left(\frac{1}{z}\right)\right) \\
& w(z)=c_{(a, b)} h\left(a \phi\left(\frac{b}{z}\right)\right),
\end{aligned}
$$

and

$$
c_{(a, b)}=\int_{0}^{a} \int_{0}^{b} D_{1} D_{2} f(\gamma(s, t)) D_{1} D_{2} \gamma(s, t) d s d t
$$

\section{Main results and proofs}

Theorem 2.1 Let $f$ and $g$ be convex and increasing functions on $[0, \infty)$ with $f(0)=0$, and let $p(s, t) \geq 0, D_{1} D_{2} p(s, t)=\frac{\partial^{2}}{\partial s \partial t} p(s, t), D_{1} D_{2} p(s, t)>0, s \in[\alpha, a], t \in[\beta, b]$ with $p$ $(s, \beta)=p(\alpha, t)=p(\alpha, \beta)=0$ and $\left.D_{1} D_{2} p(s, t)\right|_{t=\tau}=0$. Further, let $x(s, t)$ be absolutely continuous on $[\alpha, a) \times[\beta, b]$, and $x(s, \beta)=x(\alpha, t)=x(\alpha, \beta)=0$. Then, following inequality holds

$$
\begin{gathered}
\int_{\alpha}^{a} \int_{\beta}^{b} D_{1} D_{2} p(s, t) \cdot g\left(\frac{\left|D_{1} D_{2} x(s, t)\right|}{D_{1} D_{2} p(s, t)}\right) \cdot \frac{\partial}{\partial t}\left[f\left(p(s, t) \cdot g\left(\frac{|x(s, t)|}{p(s, t)}\right)\right)\right] d s d t \\
\leq f\left(\int_{\alpha}^{a} \int_{\beta}^{b} D_{1} D_{2} p(s, t) \cdot g\left(\frac{\left|D_{1} D_{2} x(s, t)\right|}{D_{1} D_{2} p(s, t)}\right) d s d t\right) .
\end{gathered}
$$

Proof Let $y(s, t)=\int_{\alpha}^{s} \int_{\beta}^{t}\left|D_{1} D_{2} x(\sigma, \tau)\right| d \sigma d \tau$ so that $D_{1} D_{2} y(s, t)=\left|D_{1} D_{2} x(s, t)\right|$ and $y(s, t) \geq|x(s, t)|$. Thus, from Jensen's integral inequality, we obtain

$$
\begin{aligned}
g\left(\frac{|x(s, t)|}{p(s, t)}\right) \leq & g\left(\frac{\gamma(s, t)}{p(s, t)}\right) \leq g\left(\frac{\int_{\alpha}^{s} \int_{\beta}^{t} D_{1} D_{2} p(\sigma, \tau) \frac{\left|D_{1} D_{2} x(\sigma, \tau)\right|}{D_{1} D_{2} p(\sigma, \tau)} d \sigma d \tau}{\int_{\alpha}^{s} \int_{\beta}^{t} D_{1} D_{2} p(\sigma, \tau) d \sigma d \tau}\right) \\
& \leq \frac{1}{p(s, t)} \int_{\alpha}^{s} \int_{\beta}^{t} D_{1} D_{2} p(\sigma, \tau) g\left(\frac{\left|D_{1} D_{2} x(\sigma, \tau)\right|}{D_{1} D_{2} p(\sigma, \tau)}\right) d \sigma d \tau .
\end{aligned}
$$

By using the inequality (2.2), we have

$$
\begin{gathered}
\int_{\alpha}^{a} \int_{\beta}^{b} D_{1} D_{2} p(s, t) \cdot g\left(\frac{\left|D_{1} D_{2} x(s, t)\right|}{D_{1} D_{2} p(s, t)}\right) \cdot \frac{\partial}{\partial t}\left[f\left(p(s, t) \cdot g\left(\frac{|x(s, t)|}{p(s, t)}\right)\right)\right] d s d t \\
\leq \int_{\alpha}^{a} \int_{\beta}^{b} D_{1} D_{2} p(s, t) \cdot g\left(\frac{D_{1} D_{2} \gamma(s, t)}{D_{1} D_{2} p(s, t)}\right) \cdot \frac{\partial}{\partial t}\left[f\left(\int_{\alpha}^{s} \int_{\beta}^{t} D_{1} D_{2} p(\sigma, \tau) \cdot g\left(\frac{D_{1} D_{2} \gamma(\sigma, \tau)}{D_{1} D_{2} p(\sigma, \tau)}\right) d \sigma d \tau\right)\right] d s d t .
\end{gathered}
$$

On the other hand

$$
\begin{gathered}
\frac{\partial^{2}}{\partial s \partial t}\left[f\left(\int_{\alpha}^{s} \int_{\beta}^{t} D_{1} D_{2} p(\sigma, \tau) \cdot g\left(\frac{D_{1} D_{2} \gamma(\sigma, \tau)}{D_{1} D_{2} p(\sigma, \tau)}\right) d \sigma d \tau\right)\right] \\
=\frac{\partial}{\partial s}\left\{\frac{\partial}{\partial t}\left[f\left(\int_{\alpha}^{s} \int_{\beta}^{t} D_{1} D_{2} p(\sigma, \tau) \cdot g\left(\frac{D_{1} D_{2} \gamma(\sigma, \tau)}{D_{1} D_{2} p(\sigma, \tau)}\right) d \sigma d \tau\right)\right] \cdot \int_{\alpha}^{s} p_{\sigma t}(\sigma, t) \cdot g\left(\frac{D_{1} D_{2} \gamma(\sigma, \tau)}{D_{1} D_{2} p(\sigma, t)}\right) d \sigma\right\} \\
=\left\{\frac{\partial^{2}}{\partial s \partial t}\left[f\left(\int_{\alpha}^{s} \int_{\beta}^{t} D_{1} D_{2} p(\sigma, \tau) \cdot g\left(\frac{D_{1} D_{2} \gamma(\sigma, \tau)}{D_{1} D_{2} p(\sigma, \tau)}\right) d \sigma d \tau\right)\right]\right\} \cdot \int_{\alpha}^{s} D_{1} D_{2} p(\sigma, t) \cdot g\left(\frac{D_{1} D_{2} \gamma(\sigma, \tau)}{p_{\sigma t}(\sigma, t)}\right) d \sigma \\
\times \int_{\beta}^{t} p_{s \tau}(s, \tau) \cdot g\left(\frac{D_{1} D_{2} \gamma(\sigma, \tau)}{D_{1} D_{2} p(s, \tau)}\right) d \tau+D_{1} D_{2} p(s, t) \cdot g\left(\frac{D_{1} D_{2} \gamma(s, t)}{D_{1} D_{2} p(s, t)}\right) \\
\times \frac{\partial}{\partial t}\left[f\left(\int_{\alpha}^{s} \int_{\beta}^{t} D_{1} D_{2} p(\sigma, \tau) \cdot g\left(\frac{D_{1} D_{2} \gamma(\sigma, \tau)}{D_{1} D_{2} p(\sigma, \tau)}\right) d \sigma d \tau\right)\right] \\
=D_{1} D_{2} p(s, t) \cdot g\left(\frac{D_{1} D_{2} \gamma(s, t)}{D_{1} D_{2} p(s, t)}\right) \cdot \frac{\partial f}{\partial t}\left[\left(\int_{\alpha}^{s} \int_{\beta}^{t} D_{1} D_{2} p(\sigma, \tau) \cdot g\left(\frac{D_{1} D_{2} \gamma(\sigma, \tau)}{D_{1} D_{2} p(\sigma, \tau)}\right) d \sigma d \tau\right)\right] .
\end{gathered}
$$


From (2.3) and (2.4), we have

$$
\begin{gathered}
\int_{\alpha}^{a} \int_{\beta}^{b} D_{1} D_{2} p(s, t) \cdot g\left(\frac{\left|D_{1} D_{2} x(s, t)\right|}{D_{1} D_{2} p(s, t)}\right) \cdot \frac{\partial}{\partial t}\left[f\left(p(s, t) \cdot g\left(\frac{|x(s, t)|}{p(s, t)}\right)\right)\right] d s d t \\
\leq \int_{\alpha}^{a} \int_{\beta}^{b} \frac{\partial^{2}}{\partial s \partial t}\left[f\left(\int_{\alpha}^{s} \int_{\beta}^{t} D_{1} D_{2} p(\sigma, \tau) \cdot g\left(\frac{D_{1} D_{2} \gamma(\sigma, \tau)}{D_{1} D_{2} p(\sigma, \tau)}\right) d \sigma d \tau\right)\right] d s d t \\
=f\left(\int_{\alpha}^{a} \int_{\beta}^{b} D_{1} D_{2} p(\sigma, \tau) \cdot g\left(\frac{D_{1} D_{2} \gamma(\sigma, \tau)}{D_{1} D_{2} p(\sigma, \tau)}\right) d \sigma d \tau\right) \\
=f\left(\int_{\alpha}^{a} \int_{\beta}^{b} D_{1} D_{2} p(s, t) \cdot g\left(\frac{\left|D_{1} D_{2} x(s, t)\right|}{D_{1} D_{2} p(s, t)}\right) d s d t\right) .
\end{gathered}
$$

This completes the proof.

Remark 2.2 Let $x(s, t)$ reduce to $s(t)$, and with suitable modifications in the proof of Theorem 2.1, then (2.1) becomes inequality (1.5) stated in Section 1.

Remark 2.3 Taking for $g(x)=x$ in (2.1), then (2.1) becomes the following inequality.

$$
\int_{\alpha}^{a} \int_{\beta}^{b}\left|D_{1} D_{2} x(s, t)\right| \cdot \frac{\partial}{\partial t}(f(|x(s, t)|)) d s d t \leq f\left(\int_{\alpha}^{a} \int_{\beta}^{b}\left|D_{1} D_{2} x(s, t)\right| d s d t\right) .
$$

Let $x(s, t)$ reduce to $s(t)$, and with suitable modifications, then (2.5) becomes inequality (1.4) stated in Section 1.

Remark 2.4 For $f(t)=t^{l+1}, l \geq 0$, the inequality (2.5) reduces to

$$
\int_{\alpha}^{a} \int_{\beta}^{b}|x(s, t)|^{l} \frac{\partial}{\partial t}(|x(s, t)|) d s d t \leq \frac{1}{l+1}\left(\int_{\alpha}^{a} \int_{\beta}^{b}\left|D_{1} D_{2} x(s, t)\right| d s d t\right)^{l+1} .
$$

In the right side of (2.6), by Hölder inequality with indices $l+1$ and $(l+1) l$, gives

$$
\int_{\alpha}^{a} \int_{\beta}^{b}|x(s, t)|^{l} \frac{\partial}{\partial t}(|x(s, t)|) d s d t \leq \frac{[(a-\alpha)(b-\beta)]^{l}}{l+1} \int_{\alpha}^{a} \int_{\beta}^{b}\left|D_{1} D_{2} x(s, t)\right|^{l+1} d s d t .
$$

Let $x(s, t)$ reduce to $s(t)$ and $\alpha=\beta=0$, then (2.7) becomes Hua's inequality (1.3) stated in Section 1.

Theorem 2.5 Assume that

(i) $f$, $g$ and $x(s, t)$ are as in Theorem 2.1,

(ii) $p(s, t)$ is increasing on $[0, a] \times[0, b]$ with $p(s, \beta)=p(\alpha, t)=p(\alpha, \beta)=0$,

(iii) $h$ is concave and increasing on $[0, \infty)$,

(iv) $\varphi(t)$ is increasing on $[0, a]$ with $\varphi(0)=0$,

(v) For $y(s, t)=\int_{0}^{s} \int_{0}^{t} D_{1} D_{2} p(\sigma, \tau) g\left(\frac{\left|D_{1} D_{2} x(\sigma, \tau)\right|}{D_{1} D_{2} p(\sigma, \tau)}\right) d \sigma d \tau$,

$$
D_{1} D_{2} f(\gamma(s, t)) D_{1} D_{2} \gamma(s, t) \cdot \phi\left(\frac{1}{D_{1} D_{2} \gamma(s, t)}\right) \leq \frac{c_{(a, b)}}{\gamma(a, b)} \cdot \phi^{\prime}\left(\frac{t}{\gamma(a, b)}\right) .
$$


Then

$$
\begin{array}{rl}
\int_{0}^{a} \int_{0}^{b} D_{1} D_{2} & f\left(p(s, t) g\left(\frac{|x(s, t)|}{p(s, t)}\right)\right) \cdot v\left(D_{1} D_{2} p(s, t) g\left(\frac{\left|D_{1} D_{2} x(s, t)\right|}{D_{1} D_{2} p(s, t)}\right)\right) d s d t \\
& \leq w\left(\int_{0}^{a} \int_{0}^{b} D_{1} D_{2} p(s, t) g\left(\frac{\left|D_{1} D_{2} x(s, t)\right|}{D_{1} D_{2} p(s, t)}\right) d s d t\right),
\end{array}
$$

where

$$
\begin{aligned}
& v(z)=z h\left(\phi\left(\frac{1}{z}\right)\right), \\
& w(z)=c_{(a, b)} h\left(a \phi\left(\frac{b}{z}\right)\right) .
\end{aligned}
$$

and

$$
c_{(a, b)}=\int_{0}^{a} \int_{0}^{b} D_{1} D_{2} f(\gamma(s, t)) D_{1} D_{2} \gamma(s, t) d s d t
$$

Proof From (2.2), we easily obtain

$$
p(s, t) g\left(\frac{|x(s, t)|}{p(s, t)}\right) \leq \int_{0}^{s} \int_{0}^{t} D_{1} D_{2} p(\sigma, \tau) g\left(\frac{\left|D_{1} D_{2} x(\sigma, \tau)\right|}{D_{1} D_{2} p(\sigma, \tau)}\right) d \sigma d \tau=\gamma(s, t) .
$$

From (2.8), (2.10-2.12) and Jensen's inequality(for concave function), hence

$$
\begin{aligned}
& \int_{0}^{a} \int_{0}^{b} D_{1} D_{2} f\left(p(s, t) g\left(\frac{|x(s, t)|}{p(s, t)}\right)\right) \cdot v\left(D_{1} D_{2} p(s, t) g\left(\frac{\left|D_{1} D_{2} x(s, t)\right|}{D_{1} D_{2} p(s, t)}\right)\right) d s d t \\
& \leq \int_{0}^{a} \int_{0}^{b} D_{1} D_{2} f(\gamma(s, t)) \cdot v\left(D_{1} D_{2} \gamma(s, t)\right) d s d t \\
& =\int_{0}^{a} \int_{0}^{b} D_{1} D_{2} f(\gamma(s, t)) D_{1} D_{2} \gamma(s, t) \cdot h\left(\phi\left(\frac{1}{D_{1} D_{2} \gamma(s, t)}\right)\right) d s d t \\
& =\frac{\int_{0}^{a} \int_{0}^{b} D_{1} D_{2} f(\gamma(s, t)) D_{1} D_{2} \gamma(s, t) \cdot h\left(\phi\left(\frac{1}{D_{1} D_{2} \gamma(s, t)}\right)\right) d s d t}{\int_{0}^{a} \int_{0}^{b} D_{1} D_{2} f(\gamma(s, t)) D_{1} D_{2} \gamma(s, t) d s d t} \\
& \times \int_{0}^{a} \int_{0}^{b} D_{1} D_{2} f(\gamma(s, t)) D_{1} D_{2} \gamma(s, t) d s d t \\
& \leq h\left(\frac{\int_{0}^{a} \int_{0}^{b} D_{1} D_{2} f(\gamma(s, t)) D_{1} D_{2} \gamma(s, t) \cdot \phi\left(\frac{1}{D_{1} D_{2} \gamma(s, t)}\right) d s d t}{\int_{0}^{a} \int_{0}^{b} D_{1} D_{2} f(\gamma(s, t)) D_{1} D_{2} \gamma(s, t) d s d t}\right) \cdot c_{(a, b)} \\
& \leq h\left(\frac{\int_{0}^{a} \int_{0}^{b} \frac{c_{(a, b)}}{\gamma(a, b)} \cdot \phi^{\prime}\left(\frac{t}{\gamma(a, b)}\right) d s d t}{c_{(a, b)}}\right) \cdot c_{(a, b)} \\
& =h\left(\frac{1}{y(a, b)} \int_{0}^{a}\left(\left.y(a, b) \phi\left(\frac{t}{y(a, b)}\right)\right|_{t=0} ^{t=b}\right) d s\right) \cdot c_{(a, b)} \\
& =h\left(a \phi\left(\frac{b}{y(a, b)}\right)\right) \cdot c_{(a, b)} \\
& =w\left(\int_{0}^{a} \int_{0}^{b} D_{1} D_{2} p(s, t) g\left(\frac{\left|D_{1} D_{2} x(s, t)\right|}{D_{1} D_{2} p(s, t)}\right) d s d t\right) .
\end{aligned}
$$


This completes the proof.

Remark 2.6 Let $x(s, t)$ reduce to $s(t)$, and with suitable modifications in the proof of Theorem 2.5 , then (2.9) becomes the following inequality:

$$
\int_{0}^{a} f^{\prime}\left(p(t) g\left(\frac{|x(t)|}{p(t)}\right)\right) \cdot v\left(p^{\prime}(t) g\left(\frac{\left|x^{\prime}(t)\right|}{p^{\prime}(t)}\right)\right) d t \leq w\left(\int_{0}^{a} p^{\prime}(t) g\left(\frac{\left|x^{\prime}(t)\right|}{p^{\prime}(t)}\right) d t\right) .
$$

The inequality has been obtained by Rozanova in [17]. For $x(t)=x_{1}(t), x_{1}^{\prime}(t)>0, x_{1}(0)=0, x(a)=b, g(t)=t, f(t)=\phi(t)=t^{2} \quad$ and $\quad h(t)=\sqrt{1+t}$, the inequality (2.13) reduces to Polya's inequality (see [17]).

Remark 2.7 Taking for $g(x)=x$ in (2.9), then (2.9) becomes the following interesting inequality.

$$
\int_{0}^{a} \int_{0}^{b} D_{1} D_{2} f(|x(s, t)|) \cdot v\left(\left|D_{1} D_{2} x(s, t)\right|\right) d s d t \leq w\left(\int_{0}^{a} \int_{0}^{b}\left|D_{1} D_{2} x(s, t)\right| d s d t\right) .
$$

\section{Acknowledgements}

The authors express their deep gratitude to the referees for their many very valuable suggestions and comments. The research of Chang-Jian Zhao was supported by National Natural Science Foundation of China (10971205), and the research of Wing-Sum Cheung was partially supported by a HKU URC grant.

\section{Author details}

'Department of Mathematics, China Jiliang University, Hangzhou 310018, China ${ }^{2}$ Department of Mathematics, The University of Hong Kong, Pokfulam Road, Hong Kong

\section{Authors' contributions}

C-JZ and W-SC jointly contributed to the main results Theorems 2.1 and 2.5. Both authors read and approved the final manuscript.

\section{Competing interests}

The authors declare that they have no competing interests.

Received: 14 March 2011 Accepted: 18 August 2011 Published: 18 August 2011

\section{References}

1. Opial, Z: Sur une inégalité. Ann Polon Math. 8, 29-32 (1960)

2. Willett, D: The existence-uniqueness theorem for an $n$-th order linear ordinary differential equation. Am Math Monthly. 75, 174-178 (1968). doi:10.2307/2315901

3. Hua, LK: On an inequality of Opial. Sci China. 14, 789-790 (1965)

4. Godunova, EK: Integral'nye neravenstva s proizvodnysi i proizvol'nymi vypuklymi funkcijami. Uc Zap Mosk Gos Ped In-ta im Lenina. 460, 58-65 (1972)

5. Rozanova, Gl: On an inequality of Maroni (Russian). Math Zametki. 2, 221-224 (1967)

6. Das, KM: An inequality similar to Opial's inequality. Proc Am Math Soc. 22, 258-261 (1969)

7. Agarwal, RP, Thandapani, E: On some new integrodifferential inequalities. Anal sti Univ "Al I Cuza" din lasi. 28, 123-126 (1982)

8. Yang, GS: A note on inequality similar to Opial inequality. Tamkang J Math. 18, 101-104 (1987)

9. Agarwal, RP, Lakshmikantham, V: Uniqueness and Nonuniqueness Criteria for Ordinary Differential Equations. World Scientific, Singapore (1993)

10. Bainov, D, Simeonov, P: Integral Inequalities and Applications. Kluwer Academic Publishers, Dordrecht (1992)

11. Li, JD: Opial-type integral inequalities involving several higher order derivatives. J Math Anal Appl. 167, 98-100 (1992). doi:10.1016/0022-247X(92)90238-9

12. Cheung, WS: On Opial-type inequalities in two variables. Aequationes Math. 38, 236-244 (1989). doi:10.1007/ BF01840008

13. Cheung, WS: Some generalized Opial-type inequalities. J Math Anal Appl. 162, 317-321 (1991). doi:10.1016/0022-247X (91)90152-P

14. Zhao, CJ, Cheung, WS: Sharp integral inequalities involving high-order partial derivatives. J Inequal Appl (2008). Article ID 571417

15. Agarwal, RP, Pang, PYH: Sharp opial-type inequalities in two variables. Appl Anal. 56(3), 227-242 (1996). doi:10.1080/ 00036819508840324

16. Agarwal, RP, Pang, PYH: Opial Inequalities with Applications in Differential and Difference Equations. Kluwer Academic Publishers, Dordrecht (1995) 
17. Rozanova, Gl: Ob odnom integral'nom neravenstve, svjazannom s neravenstvom Polia. Izvestija Vyss Ucebn, Zaved Mat. $125,75-80(1975)$

doi:10.1186/1029-242X-2011-33

Cite this article as: Zhao and Cheung: On improvements of the Rozanova's inequality. Journal of Inequalities and Applications 2011 2011:33.

Submit your manuscript to a SpringerOpen ${ }^{\odot}$ journal and benefit from:

- Convenient online submission

- Rigorous peer review

- Immediate publication on acceptance

- Open access: articles freely available online

- High visibility within the field

- Retaining the copyright to your article

Submit your next manuscript at $\gg$ springeropen.com 